\title{
Aesthetics of Resolution. A postdisciplinary approach to countering the technocapitalist black box
}

\section{SIGRADI2018 TECHNOPOLITICAS \\ xxii congresso da sociedade iberoamericana de gráfica digital 22th conference of the iberoamerican society of digital graphics 07|08|09|novembro|2018 iau usp | são carlos | sp br}

\author{
Gerald Nestler, PhD \\ Independent artist | Austria | mail@geraldnestler.net
}

\begin{abstract}
Visibility and knowledge are based on access to information. We usually consider this as either a question of collecting new or examining existing data. However, the term "black box society" (Pasquale) points to a situation in which data are deliberately concealed, enabling complex processes of technocapitalist exploitation. Manufacturing information asymmetry and noise have become effective tools to gain competitive advantage across all levels of life. This text argues that adverse technopolitical schemes can be addressed with an aesthetics of resolution and with the figure of the renegade, an expert who makes the black box speak from inside.
\end{abstract}

Keywords: Aesthetics; Black box automation; Big data; Finance; Information asymmetry; Resolution; Renegade
INTRODUCTION

\section{CAPITULATION AUTOMATED - RESOLUTION AND DISSOLUTION BEYOND VISIBILITY}

"99 per cent of finance doesn't know how the stock market works." - Haim Bodek

On May 6, 2010, bots played havoc among financial market centers causing mayhem in less than five minutes. The Flash Crash, as it has become known, went viral as the biggest one-day decline in the history of financial markets. During the rapid slump, the Dow Jones Industrial Average plunged by about 1,000 points - nine per cent of its total value - only to recover most of its losses in the next twenty minutes. CNBC Live, initially covering the political stalemate of the Greek austerity crisis and the ensuing protests in the streets of Athens, shifted immediately to the trading floor of the New York stock exchange: "What the heck is going on down there? ... I don't know... this is fear, this is capitulation."

The Flash Crash was a watershed event in financial markets. Algorithmic trading had taken center-stage. Human traders lost their bearings in the event. A livebroadcast for professional traders commented: "This will blow people out in a big way like you won't believe." ${ }^{2}$ Technically, capitulation means panic selling due to pessimism and resignation. But apart from financial losses, the term "capitulation" implies a liquidation of visibility in the sense of unmediated human perception and collective resolution. Hence, the broadcast highlighted the relevance of political economy today. What informs such potent noise without leaving much of a trace? And how does it affect us?

1 CNBC news coverage of the Flash Crash. Online: https://www.youtube.com/watch?v=Bnc9jR2WDgo, last accessed June 23, 2018.

2 Ben Lichtenstein, voice of the CME S\&P futures pit, in his live-coverage of the Flash Crash, https://vimeo.com/103128278 (10:42 min).
The debate that ensued in the aftermath of the event for the first time in financial market history pitted those who uphold the generally accepted opinion, which blames human error, against a dissenting opinion, which hold algorithmic trading and automation accountable. As a collateral, high-frequency trading (HFT) came to public attention, as journalists and bloggers picked up on the topic. ${ }^{3}$ Eventually, it appeared as if the financial markets were at the mercy of quants and developers who tuned hardware, tweaked infrastructure and coded algorithms that drive automated speculations. The financial expert and whistleblower Haim Bodek speaks of "avalanching" to describe the real time violence by which HFT orders impact markets. A massive meltdown that would dwarf the Flash Crash is an event whose probability is far above zero today.

HFT achieved unparalleled market shares (up to 80 per cent of U.S. stock transactions in 2012) due to high trading volume necessary to deliver profits. However, HFT as the characteristic feature of contemporary finance is often exaggerated. Revenues are relatively small compared to the total market. ${ }^{4}$ Recently, market share has dropped due to hypercompetition and infrastructure costs that accrue in the effort to stay competitive in markets with diminishing bid-ask spreads (a result of competition). ${ }^{5}$ However, automation and algorithms have, apart from exploiting „predictive structures” (MacKenzie $(2016,6)$, ushered in a new trading paradigm: direct interception into the pricing mechanism relying on speed (or, technically, low latency).

3 See Wikipedia entry "2010 Flash Crash" for a list of noted contributions.

4 Revenues in the sector were at USD 7.2bn in 2009 but diminished to USD 1.3bn in 2014.

5 See, e.g., a Deutsche Bank report: https://www.dbresearch.com/PROD/DBR_INTERNET_ENPROD/PROD0000000000406105/Highfrequency_trading\%3A_Reaching_the_limits.pdf 


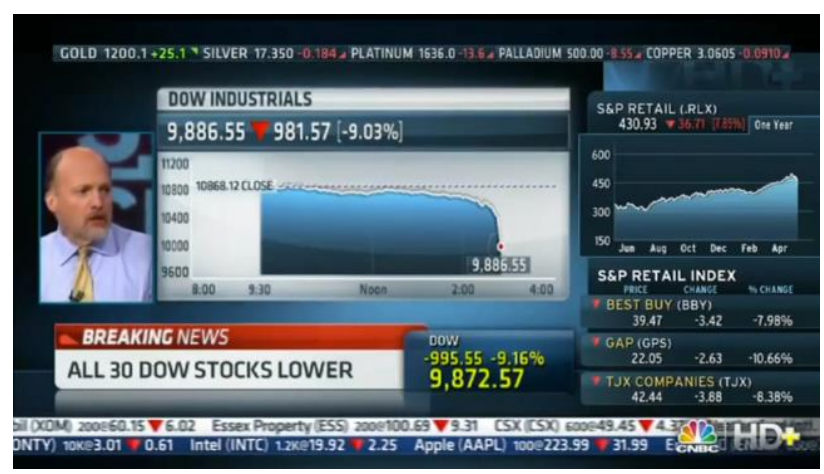

Figure 1: CNBC live coverage, Flash Crash Capitulation, 2010

With competition intensifying in this segment, too, what appeared are schemes that distort competition directly on the level of market infrastructure (for instance, the socalled order type controversy). As will be shown below, some market centers supported (and apparently still do) "regulation arbitrage:" with the growing number of exchanges - profit-seeking corporations fighting for market share, rather than public institutions - the exploitation of regulation asymmetries has become thriving business for traders in the know (which are frequently shareholders of these very market centers). Market activity increasingly borders on, or is already on the paths of, illegality.

In order to examine the consequences of HFT I will resort to the term "resolution" and its relation to immediacy as visibility. I will first look at two diverging interpretations of the Flash Crash 2010 to delineate the debate and the issues relevant for HFT and its regulation, before I enter into an examination of what seem to me the most consequential implications of quantitative trading that shape the core of this article. My interest is not in resolving which of the two positions holds true - both the official investigation by the joint CFTC/ SEC report and the analysis by the financial data provider Nanex are controversial and received criticism. Instead, I focus on the "visibility conditions" (how they relate to immediacy) and the resolution philosophy that informs them.

In brief, my proposition is threefold: Firstly, and based on my reading of the Flash Crash and financial automation, in the current technological and legal frameworks algorithmic trading is incentivized (mainly by technological innovation and by order types that are offered by profitseeking exchange places, and compound competitive advantage). Transparency rules $^{6}$ have less weight than proprietary rights; individual, sectoral and public interests are to a large extent mutually incompatible. This conflict, which I argue is pervasive in all data fields, is due to technological, legal as well as philosophical reasons and their interrelations, a fact that transcends finance into other forms of automation. The complexity of automation is not a matter of any one of these conditions but a result of their interaction. Hence, establishing visibility is in this instance a question of developing resolution tools that deliver insight. I propose the term "aesthetic of resolution" as a method to analyze black box automation. ${ }^{7}$ As I will

6 In this context, transparency does not only refer to data access but also to law and regulation.

7 Technically, black box refers to systems that feed inputs and produce outputs without disclosing their inner workings (which argue finally, rather than attempting to penetrate the black box from outside - and here I am referring not only to finance but to black boxes in general - opacity dissolves into transparency only by a shift of mentality by which aesthetics of resolution as a techno-philosophical concept turn into a politics of resolution. This shift is based on the necessity that knowledge transpires from inside the black box. It rests on what I term the figure of the renegade the notion of resolution is linked to the ambivalent figure of the informant, the whistleblower - a traitor for her field but an educator of the general public.

\section{THE FLASH CRASH - RESOLUTION IN MICROTIME}

"A distributed system is one in which the failure of a computer you didn't even know existed can render your own computer unusable." - Leslie Lamport

The investigation of the Flash Crash resulted in a joint official report by the United States regulatory authorities, the SEC and the CFTC (2010). It was published a few months after the incident and put the blame on human trading. But a contrasting analysis of the event conducted by a small financial data provider claimed that the crash was in fact caused by orders executed automatically by algorithms. Nanex LLC records trading data and was thus in the position to examine the event on their own account.

The official report was based on material made available by exchanges and market participants, which usually has a resolution of one-minute trading intervals. Such dataset might have been adequate to analyze trading activities before the ascent of HFT, but today "in the blink of an eye, the market moves what used to take humans thirty minutes" (Eric Hunsader, founder of NANEX, in: Taggart, 2010). This quote by the founder of Nanex illustrates the sheer pointlessness of scrutinizing market activity above the transaction frequency of the fastest traders. Their professional experience with market data allowed Nanex to intuitively escape the trap of one-minute resolution, which in our case conceals more than it reveals. ${ }^{8}$ They realized that conventional market data records did not show any material traces of what might have initiated the rupture that tore the intricate fabric of market prices. Hence, they delved deeper into the 'abyss' of micro-time to look at fractions of a second. Step-by-step, they enhanced the temporal resolution, and eventually, at dizzying depths of time, the material traces of the Flash Crash came into view.

When Nanex made a strike of market activity far below the threshold of perception, what they 'saw' at first glance looked like a glitch. But what emerged from the forensic analysis were the imprints of an elaborate scheme. They had encountered information in a realm that was deemed to only emit noise if anything. ${ }^{9}$ As Eric Hunsader stated:

might be unknown). In finance, it denotes computer-based trading systems that apply algorithms to trade financial products automatically.

8 "We think it's important to note that the SEC claimed there is no value to be gained from looking at data in time resolutions under a second 'because it is just noise'. We strongly disagree." See: http://www.nanex.net/aqck2/3522.html

9 Noise as opposed to signal is the term for random information in information theory. As financial markets are constructed as information markets (both in the Hayekian sense 
"The SEC/CFTC analysts clearly didn't have the dataset to do it in the first place. One-minute snapshot data, you can't tell what happened inside of that minute. We didn't really see the relationship between the trades and the quote rates until we went under a second" (Nanex, 2010). Their final statement was unambiguous: "High Frequency Trading caused the Flash Crash. Of this, we are sure."10

As mentioned above, I won't address the truth claims of both the official report and Nanex. My main concern is the discrepancy between the material traces and their consequences. I will focus on Nanex as provider of a set of forensic resolutions on the material data of the Flash Crash, which raises the question of how resolution techniques operate as regards visualization (making tools that enhance perception and render material evidence); evaluative measuring (computation of sequences and relations); and knowledge production (analysis and interpretation). Even though high-resolution 'telescoping' offers glimpses into financial microtime, attribution and solution - decisive elements of the semantic field of the term resolution - rest beyond the capacity of Nanex or any third party (market participants and the general public). The question as regards the actuator(s) of the Flash Crash has still not been resolved. ${ }^{11}$

\section{REPERFORMATIVE FORENSICS IN A HYPERCOMPETITIVE ENVIRONMENT}

"All consciousness is a matter of threshold." - Gilles Deleuze

An investigation into the complexity of market interplay is not only confronted with one or several black boxes but with the meta-black box of the market per se. It encompasses the entire system in its complexity, including but not restricted to brokers, traders (in the broadest term), market makers and centers. As the former HFT trader David Lauer remarks: "The markets and the interplay in the industry between all these firms with all these very complicated and complex technology systems and how they interact makes the entire system of exchanges, high-frequency, brokers and the interaction between the technology, it makes it a complex system. There is no cause and effect [...] What caused the Flash Crash is a nonsense question. [...] if you were to replay the same sequence of events, identically, there's no guarantee that it will cause a Flash Crash again. That's the nature of complex systems" (Meerman, 2013).

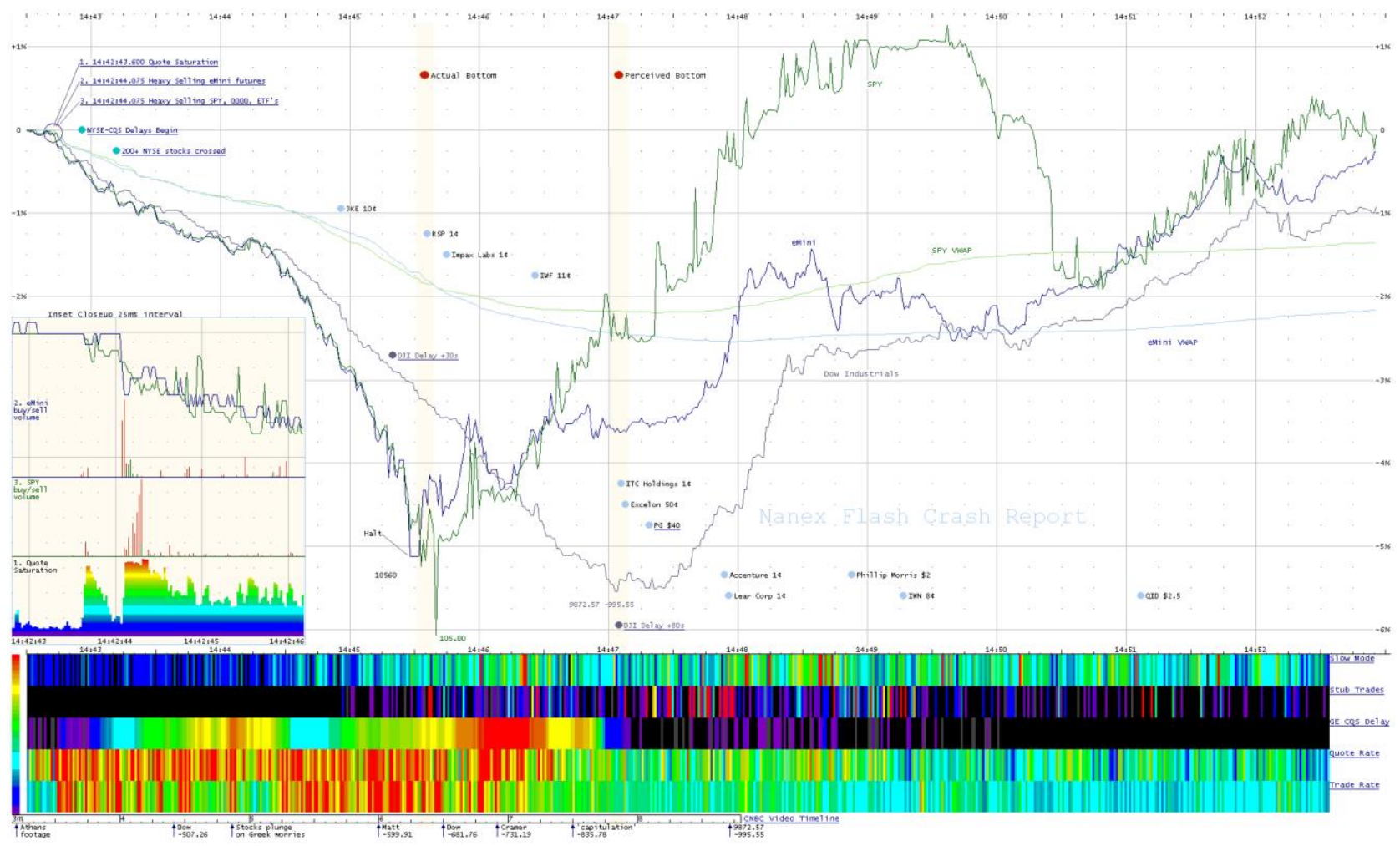

Figure 2: Flash Crash analysis, courtesy Nanex LLC

and cybernetics), noise is a constituent element of trading. Following Black $(1986,530)$ we can define it as the ubiquitous other of information: "Noise makes financial markets possible, but also makes them imperfect."

10 Despite the evidence of trades, evidence on the perpetrator is impenetrable, as the law protects proprietary data and its source.

11 This essay is also not concerned with the exposure of an alleged culprit in 2016. 
After failing to attribute motive and blame, Nanex changed their investigative strategy to mixed forensic analysis and witness review. They asked the mutual fund Waddell \& Reed - the party blamed by not identified by the official report - to grant access to their trading data. it is most plausible that in accordance with the capitalist proprietary regime the fund would have declined disclosure had they not been blamed. But by the time Waddell \& Reed had a vested interest in clearing their name. The incorporation of the proprietary source code allowed Nanex to classify the data of a specific address. Their analysis relied on an apparatus

that pairs four quantitative frameworks in an effort to deliver sufficient approximation to the trading operations: Nanex's extensive archive of financial data; quantitative analysis; custom-made, adaptive resolution devices that power the investigation of data sets; and the algorithmic trading data from the financial black box (including the execution algorithm of Barclay Capital Inc., their broker). ${ }^{12}$ This framework led Nanex to deviate from the official narrative. Although their interpretation is controversial (algo traders, for instance, hailed the official report), it brought the cybernetic regime of HFT to light.

A paper co-authored by members of the official investigation maintains the narrative that "HFT did not cause the Flash Crash, but contributed to extraordinary market volatility experienced on May 6, 2010. [...] high frequency trading contributes to flash-crash-type events by exploiting short-lived imbalances in market conditions" (Kirilenko et al., 2014, 2). The authors detect immediacy as problematic, as it is exacerbated by HFT to its own benefit. "Because advanced trading technology can be deployed with little alteration across many automated markets, the cost of providing intermediation services per market has fallen drastically. As a result, the supply of immediacy provided by the HFTs has skyrocketed. [...] the benefits of immediacy accrue disproportionally to those who possess the technology to take advantage of it. As a result, HFTs have become the main beneficiaries of immediacy, using it not only to lower their adverse selection costs, but also to take advantage of the customers who dislike adverse selection and do not have the technology to be able to trade as quickly as they would like to. [...] Consequently, HFTs can both increase their demand for immediacy and decrease their supply of immediacy just ahead of any slower immediacy-seeking customer." (Kirilenko et al., 3).

Immediacy, l'd argue, defines visibility as a performative issue. In relation to market activity - especially in, but not restricted to, times of high volatility - immediacy equals visibility: immediacy is technological visibility constructed by resolution techniques. Developers in the algorithmic trading space increase obscurity within the entire playing field by narrowing, if not modifying, the field of visibility (of the order book, to be precise). This instance is the most current in a row of performative revolutions in finance that started with the displacement of (human) floor traders by quants (the framing of finance by Black Scholes Merton (BSM); the quantification of the volatility curve in the

12 See, for example:
http://www.reuters.com/article/flashcrash-barclaysidUSN2219178020101022 pricing regime; which goes hand in hand with shifts between cost centers and profit centers. ${ }^{13}$

When we accept immediacy as a form of visibility in microtime - engineered by resolution techniques that both enhance the visual field and act upon it (and obscuring it for those lacking the means) - we can adopt Michel Callon and Donald MacKenzie's theory of performativity. While MacKenzie focuses on a different realm of financial market activity - precisely derivatives trading and the BSM model, and hence the profit model of finance that was challenged by low latency trading applications Callon (2006) offers a wider view on performativity: „My thesis is that both the natural and life sciences, along with the social sciences, contribute towards enacting the realities that they describe." The black box as a scientific apparatus has conquered finance and constructs its reality in a similar way as economic theories acted on it.

In the light of recurring flash crashes and avalanches, MacKenzie's "counterperformativity" ${ }^{14}$ seems to highlight this fact even more robustly: Whereas the notion of a selffulfilling prophecy explains success or failure in terms of beliefs only, that of performativity goes beyond human minds and deploys all the materialities comprising the socio-technical agencements that constitute the world in which these agents are plunged: performativity leaves open the possibility of events that might refute, or even happen independently of, what humans believe or think.

Due to the complexity described by Lauer, the market cannot simply be "captured" in all its immediacy and "replayed" like a film. ${ }^{15}$ The vision-enhancing sensors, which detect time-blurred traces and mark discriminations in a complex environment, deliver information from noise. It has to be unearthed and then resolved in a separate stage. Thus, a forensic analysis is neither fully embodied nor defined by the abstract representations of data traffic. Rather, the analysis is situated, i.e. constructed, inbetween the juncture of performance as the actual presence of an event (exemplified by the occurrence of the Flash Crash); performative analysis as providing "visual collateral" of a "re-animation" of the original obscured presence after the fact; and beyond market activity per se and thus beyond MacKenzie's use of the term "counterperformativity," as the effect of a renegade act disclosing material otherwise under non-disclosure and as a consequence of "capitulation."16

13 I owe this assessment to the HFT expert and whistleblower Haim Bodek with whom I work on a cartography of algorithmic trading.

14 For more on the term "counterperformativity," see MacKenzie $(2006,19)$.

15 Jon Roffe $(2015,71)$ emphasizes the „evental character of price [...], for, strictly speaking, no price can ever be repeated. This is because any given price is recorded on a surface and in this way changes it. To repeat the same price - where price is now grasped at the moment of its advent - can never have the same effect on the market surface itself."

16 The performative setting of Nanex's analysis might have influenced its outcome counter-performatively in the sense that analysis constructs findings and solutions. 


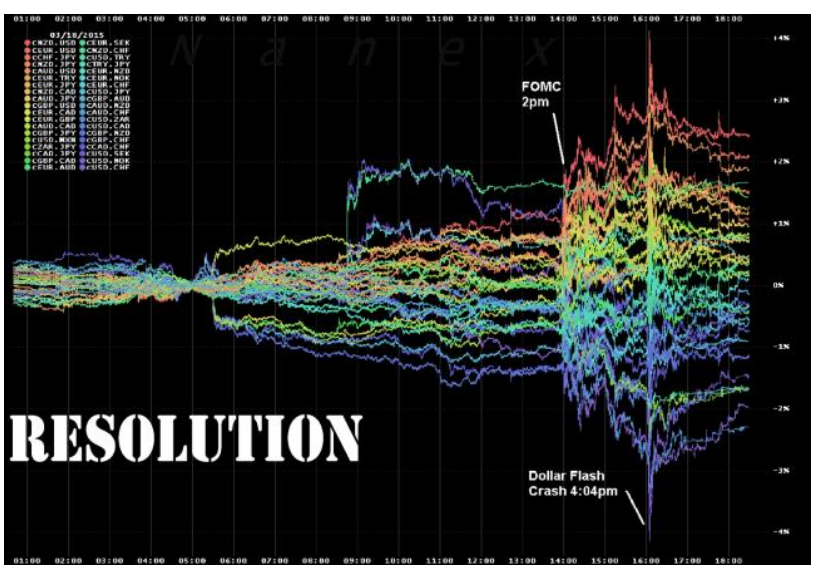

Figure 3: "2015 Dollar-Flash Crash data burst, courtesy Nanex.

We can now outline a sharper distinction, which will help us to grasp what is at play in the forensic documentation and evaluation apparatus. Artificial sense organs reach into deep time by increasing the resolution bandwidth in order to revisit the otherwise insensible "scene of the crime." The analysis is thus an intricate and extensive cybernetic undertaking characterized by a process of remapping, re-modeling, re-visioning, and re-narrating a specific black box past that happened at near-light speed-a performance ex post that was the occurrence of a future event. As this approach re-enacts the performance of the event, the methodology can be specified as a reperformance. The technological, calculative aspect of sifting data for evidence - enacting the reperformance - becomes explicit in the sheer enormity of the material Nanex examined: "May 6th had approximately 7.6 billion [...] records. We generated over 4,500 datasets and over 1,200 charts before uncovering what we believe precipitated the swift 600-point drop [...]. In generating these data sets we have also developed several proprietary applications that identify the conditions described in real time or for historical analysis." ${ }^{17}$

Only rigorous research into the deeper, imperceptible strata of microscopic time reveals the material matrix. Such excavation elucidates an inversion of time from Chronos to Kairos from a chronological interpretation (replay) of pricing to one of intense event time (real time). Methodologically, it inverts the relation between time and space: while the common notion of archaeology entails entering into concrete and thick space cautiously (as when employing technologies of surveying, probing, and classifying), in order to extract the material witness (a truth) of a former era, archaeology of finance is forensics of performance in the future. It probes the imperceptible materiality of time becoming. It detects patterns, recovers artifacts whose existence is derived from financial models and built on technological miniaturization and automation, aligned with the politics of securing, excluding, and enclosing. Research is applied to an infrastructure that 'makes happen' in which "the concept of performativity has led to the replacement of the concept of truth (or nontruth) by that of success or failure" (Callon, 2006, 13). This time around, though, it is not economic volatility modeling (i.e. hedging risk) but latency and order book penetration (i.e. the technological pursuit of riskless profits).

17 Nanex, see:

http://www.nanex.net/20100506/FlashCrashAnalysis_About.html
The Flash Crash narrative unfolds in the extended realm of trading bandwidth and the reduction of profit margins in which the technopolitical regime of success/failure becomes apparent via exclusion/inclusion, prioritizing the algorithmic aesthesis of an elite of HFT traders. For Manoi Narang (2014, 18-19), a former champion of HFT, the field's competitive ecology is synonymous with other technological applications: "many micro-industries experience an initial phase of immense profitability, which in turn attracts a great many new participants. These new participants drive up competition, which in turn causes profit margins to diminish. Eventually, so many participants are competing that margins can turn negative. Haim Bodek, however, whose HFT hedge fund went bankrupt due to "order flow information asymmetries," gives a darker view of HFT's "noisy" ecology: "There's basically interaction in the market. This firm knows how this works here, they know this practice works over there, and they're able to get to $15 \%$ or $20 \%$ of the market because they know that - and that's the only reason they can get there [...] you have this efficient market and certain regulators say it's great, it's only a penny wide, the customer gets the best price. And now l'm telling you, you can have a firm with $20 \%$ of the market and the rules change a little bit and some transparency happens and they collapse to $3 \%$. So, what's my epiphany? I basically believe that individuals running large trading companies, cannot actually tolerate a zero-profit margin environment. We will find ways around that situation. We will cheat. We will manufacture situations. We will undermine infrastructure" (in: Nestler, 2014). ${ }^{18}$

Below the radar of state agencies established to regulate market activity, corporate self-interest created an even deeper level of incorporation programmed into automated trading as the "genetic" code of a new breed of agency in the market system. Mathematical models and algorithms revolutionized the logistic infrastructure of exchanges and displaced the trading pit and its market makers (human traders known as "specialists" or "locals") in favor of faster execution rates and smaller spreads. Algorithmic traders instituted an arena around and between matching engines where they intervene without human intermediaries. Inigo Wilkins and Bogdan Dragos (2013) argue that "algorithms are no longer tools, but they are active in analyzing economic data, translating it into relevant information and producing trading orders." J. Doyne Farmer (2013), a former financial engineer and co-director of the program on complexity economics at Oxford's Institute for New Economic Thinking, notes, "under/price-time priority auction there is a huge advantage to speed." What emerged is a hypercompetitive environment that tweaks rules and infrastructures. It 'skews' the smile problem from the surface of derivatives pricing to the immediacy of latency volatility - a term I propose for the inconsistencies (rather than uncertainties) in a hypercompetitive trading environment, in which developers have replaced quants as the leaders of innovation. Even if there is an absolute limit to speed, the operable spaces of time between human perception and algorithmic reaction time are cosmic, to say the least. A divide of response time has opened up, the gaping but invisible abyss of latency: a new class of resolution enclosures and scales - and

18 A relation seems to exist between zero spread and zero marginal utility and corporate profit extraction by manufacturing monopolies.

5 
hence knowledge - has found the means to effectively hide its machinations from less immediate competitors. If derivative finance and its abstract models were (and still are) a mystery to the public at large, automated trading has escalated finance beyond all recognition. Here, Gottfried Wilhelm Leibniz's notion of apperception ceases to be a conception of conscious experience emerging from small, unconscious perceptions. The myriads of mathematically constructed small perceptions (of which these camera-engines are not at all "unconscious") define a virtual field of machine apperception where those who do not command the latest cyborg infrastructure are captured or blocked. Information asymmetry gains traction on the level of systemic visibility. Financial market architecture with its proprietary logistics is a black box not only with regard to the parameters of official inquest, but also in terms of knowability and objectivity in general (and beyond algorithmic trading proper). What the highfrequency black box emits is not information but noise. Such technowledge (a term I use to distinguish bot-coded acquisition of knowledge) exerts influence on the industry, but of necessity also incapacitates the public forum as a whole. Quantitative speech translates into algorithmic violence, invisible and insensible, built on performances that are real but unrecognizable (fictitious capital not in the sense of it being insubstantial but in the sense of applying tricks and asymmetries). Noise exceeds the category of information theory towards the asymmetric other that is not detected by the majority of market participants because it is not a signal in the sense of communication.

In the ever-denser, hypercompetitive environment of narrowing spreads only the ruthless survive. Noise is a guerilla tactics that stops at nothing in its 'pursuit' for profit. ${ }^{19}$ It is not simply a tool; it is a weapon of counterinformation that injures without directly inflicting the feeling of pain; a powerful and disruptive "rhythual," to add the layer of the 'immediated' frequency of algorithmic speech to Judith Butler's $(1997,159)$ performative "ritual": "The performative needs to be rethought not only as an act that an official language-user wields in order to implement already authorized effects, but precisely as social rhythual' [original: ritual], as one of the very "modalities of practices [that] are powerful and hard to resist precisely because they are silent and insidious, insistent and insinuating:' When we say that an insult strikes like a blow, we imply that our bodies are injured by such speech."

The most cunning insults are indirect. Fisher Black, in his seminal text succinctly entitled Noise (1986, 529), holds that "noise is information that hasn't arrived yet." When we accept, as evidence has shown, that low latency trading is defined by speed advantage, we must ascertain a bifurcation that goes far beyond competitive advantage in Hayekian information markets: Those who do not command the automated rhythual of micro-time face noise as the "silent and insidious" other of information; they cannot perform equally and thus cannot partake in "that reiterative power of [market] discourse to produce the phenomena that it regulates and constrains," to adopt

19 Haim Bodek's whistleblowing proves that exchanges are partners in crime. See, for example: http://www.wsj.com/articles/direct-edge-exchanges-to-pay-14million-penalty-over-order-type-descriptions-1421082603.
Butler's linguistic reading of performativity to the fitting speculative speech of financial markets (1993, xii). ${ }^{20}$ Insult as information asymmetry turns into the violence of noise asymmetry - a limitation of visibility that forms the space in which the reiteration of algo speech becomes the dominant language; an avalanching of "volatility created by circulatory forces so as to preserve and restore [their] liquidity" (Li Puma, 2016, 51). But such asymmetry plays out on the systemic level of platform capitalism, rather than between individual entities - corporate noiseing of information is manufactured in financial markets as well as other data fields. It is therefore a question of the politics of regulation and the law. What we are confronted with are schemes that benefit from exploiting regulation arbitrage, i.e. regulatory and legal overlaps or gaps.

\section{AESTHETICS OF RESOLUTION}

"Performativity is not about creating but about making happen." -Michel Callon

The success of HFT strategies and technologies is a convincing example of a shift in which performance implies an ontology that flattens the relations between human and non-human actors to the point of the annihilation of the former. Here, flattened ontology is not an ontology in which all are (approximately) equal, but one in which a paradigm prevails by reconfiguring nodes and connections. As soon as its reign stabilizes, hierarchies solidify, albeit in very volatile, metastable fashions. Automated markets are a consequential step in the techno-quantification of markets - including the data industry, which is market-driven and financialized (and it needs mentioning that derivatives are paradigmatic metadata!) - where technology as technowledge forms a new medium. N Katherine Hayles $(1999,13)$ reminds us, "for information to exist, it must always be instantiated in a medium." Bots act (but not always interact) on the infrastructure. To paraphrase Marshall McLuhan, they 'massage' 21 an electronic ,body' whose market environment they reconfigure into an algorithmic space. At the center of HFT are developers that speed up transactions to the level of microseconds. Profit is not primarily being sought by dynamic hedging in an uncertain environment. HFT is less engaged with implied volatility and the pricing of derivatives. Hence, quants -masters of the universe not long ago - are being replaced by big data systems. ${ }^{22}$ In this technosphere, the 'core' - the order book - is exploited directly with high volume to amass small but (relatively) riskless profits by reading, interfering and controlling matching engine signals. The production of risk - the fundamental mode of production of finance as it engages with the future - turns into an operational hazard; optimizing infrastructure, hardware and code, rather than mathematically scrutinizing pockets of uncertainty. And while the complex algorithms of derivative trading recalibrate all the prices to reengineer

20 Arjun Appadurai previously referred to Butler (2016, 111): "[She] introduced the idea of what I now refer to as retroperformativity, which allows us to see that ritual can be regarded as a framework for the co-staging of uncertainty and certainty in social life."

21 "Massaging" used in a similarly playful way as McLuhan (1964) in "the medium is the message:" The medium shapes "the scale and form of human association and action." I extend this to the algorithmic realm and the effects of bots on individual and political bodies.

22 See, for example: http://www.nytimes.com/2016/02/28/magazine/the-robots-arecoming-for-wall-street.html 
the derivative universe, HFT algos are simple in order to facilitate low latency interaction; massive amounts of data are analyzed, but trading logistics are streamlined to happen in a flash.

As regards the technological incorporation of financial markets today and their infrastructure, the principle resolution threshold is the visibility of the order book ideally, information is visible to and can instantly be acted upon by all market participants. Regulation National Market System (RegNMS) was enacted to establish such a market ideal but 'instantly' exploited by execution rates faster than price consolidation. The crucial term for realtime action is "instant", as it opens up to the whole gamut of technowledge that constantly redefines latency / speed horizons - and therefore the increment of an actionable instant as well as the constriction of immediate visibility to resolution machines. The HFT trader and whistleblower Haim Bodek ascribes the "cannibalistic" acceleration to competitive advantage: "Since 2007, we saw compression in the algorithm trading space where the profit margins approached near zero. And I am part of that problem. I ran my firm specifically to tighten up markets. We sometimes call that the race to the bottom in the business. [...] "If I can make a near-risk-free fraction of a cent and even if the whole day would have demanded a little bit more, I'm happy to do that now even if we barely make a profit because I'm basically taking away the opportunity for someone else to make a profit. [...] The strategy, which many of the algorithm trading firms did, was basically market share and just bring it to a place where our competition couldn't handle it" (in: Nestler, 2014, 14:20).

As the financial engineer and philosopher Elie Ayache points out, however, the scientific paradigm behind quantitative trading and automation hasn't changed - and thus implicitly refutes Rishi K. Narang's assertion (2015, xi) that "quantitative trading can be defined as the systematic implementation of trading strategies that human beings create through rigorous research." To Ayache, automated trading is not a new scientific method (an achievement he reserves for the volatility smile problem) but a new wave of exploitation within the probability paradigm and its futile scales $(2015,52)$ : "The market is the only place where the qualitative absolute event, the one that is irreducible to measure and scale and probability, finds quantitative expression, in a material medium borne by numbers, or rather prices. The market is quantitative history. One should keep in mind this contradiction in terms: one should remain aware that the historical event is incalculable and unquantifiable because it precedes any scale; and then understand the extraordinary nature of price (and of its medium: the market) as the quantification of that unquantifiability. This is why the market is truly the technology of the future. You have to realize that price is not a number. Quantifying the event (translating it into numbers) is impossible; yet the market is such a translation, precisely in so far as it takes place outside of possibility. 'Quantitative history' does not mean that the event is being forced into the mold of numbers. Rather, a quantity, a number of an extraordinary nature, has been found such that history can be quantified." In a nutshell, for Ayache the market is real, whereas probability is not. ${ }^{23}$ To his mind, the hard

23 Ayache's insistence on finance as a body constituted by human traders in the trading pit evokes Hayles (1999, 13): "If we can capture the Form of ones and zeros in a nonbiological problem of the volatility smile has not been resolved, nor has it been tackled in algorithmic trading on the supposition that the (option) pricing technology (BSM reversed) works. Algorithmic trading accelerates the exploitation of an old paradigm ${ }^{24}$ materially embedded in the computer-powered calculative evaluation of massive data sets. Predication machines attempt to evade their unpredictable contingent event by trading in fractions of a second. ${ }^{25}$ They reorganize the market to an extraction of price from big data. This performative paradigm exploits a future it doesn't know and doesn't need to know as it meets it immediately. The production of risk as a technology to deal with volatility - a potentially massive concept for complex societies and their needs and desires - complexifies price, but without producing a present in which the latter is translated back to value. Rather, it produces massive volatilities in the social realm. Resolution dissolves into leveraged power.

\section{THE TERM RESOLUTION AND ITS SEMANTIC FIELD}

"Noise crashes within as well as without." -N. Katherine Hayles

The notion of resolution involves technologies that engineer thinking and affecting, orient attractions and forge applications. Resolution is not restricted to technical appropriation, such as a device for perceiving (previously undiscovered worlds), a visualization tool, the setting of a laboratory, big data evaluations or the like. Neither is it only a cultural technique of conciliation and consultation to craft compromise and compensation. It is rather a basic category, though not uncharged with ideologies. An instrument of power, it inspired revolutions and served restorations. Its trajectory is towards openings and new perspectives but at the same time it can also be reversed to map the scales of new hierarchies. Fundamentally, however, resolution initializes new layers of thought that move from surface to surface in a connective, interrelating and unbiased way (initially a flat ontology) that erupt in new visions and knowledge. In such a post-disciplinary arena of research, philosophy and art are natural allies.

Resolution apparatuses are instrumental for developing tools and methodologies. They provide us with significant and relevant meaning in a technological as well as political sense but also produce competitive advantage when commodified. As such, resolution is one of information capitalism's cardinal means for producing attraction, evaluation and appreciation. This is due to the fact that it is about commensurable relations and associations (pricing), or, as Bruno Latour (1993,161) says about actors: "Nothing is by itself ordered or disordered, unique or multiple, homogeneous or heterogeneous, fluid or inert, human or inhuman, useful or useless. Never by itself, but always by others." Its productive feature of profit maximization is an inheritance

medium - say, on a computer disk - why do we need the body's superfluous flesh?"

24 How this plays out in financial corporations was shown by Ho (2009).

25 E.g. Knight Capital bankruptcy as black box event. On August 1, 2012, the HFT trader lost over 400 million Dollars in 30 minutes due to a technical error. "The glitch led to 4 million extra trades in 550 million shares that would not have existed otherwise" (Nanex). 
from post-Fordist operations (e.g. while a digital camera contains the full scope of its resolution capacity the price paid determines which resolution is unlocked; this is not simply a technique to control access and commercial interests but the sign(ature) of the capitalist order). Here, the semantic openness of the term resolution that spans from visualization to knowledge to decision is enclosed technologically, semantically and socially. Here, its intrinsically flat ontology as a communicative ensemble is breached, broken, corrupted, redirected, and stratified: The black box is a machine that exploits resolution through the whole gamut of the latter's semantic potentials. It inserts hierarchies. However, resolution techniques are a performative power and therefore never pure, impeccable and flawless. There are glitches, inconsistencies and noise that escape the probabilistic contraption, in consumer products as well as in finance. Human-technological entanglements give rise to new forms of (volatile) collectives. Hayles $(1999,29)$ general thesis comes to mind: "[t]he contemporary pressure toward dematerialization, understood as an epistemic shift toward pattern / randomness and away from presence / absence, affects human and textual bodies on two levels at once, as a change in the body (the material substrate) and as a change in the message (the codes of representation)."

While accounts of financial practice up to the 2000s, such as Mackenzie's, are concerned with "bodies" (physical and mental ones as well as devices) and their "messaging" operations (extended and augmented), automated trading conglomerations are replacing human market interaction and its 'trivial' resolution apparatuses for algorithmic high-resolution market making. Technology made it imperative to automate, control volume and reduce latency, in order to stay in the game - not only for small 'boutique' hedge funds but also for big players like investment banks. These massive real time calculations expand beyond the financial field into other black box operations that fold in new scales and hierarchies and new entanglements. Such information asymmetry - and the black box is an engine set up to produce and sustain information asymmetry - conquers the whole gamut of the semantic potentiality of resolution. It has the distinct 'advantage' that it constructs the future on a probabilistic trajectory: for the 'uninitiated,' it feels as if events happen contingently. Hence, noise and information are the two faces of a violent scheme that acts under the veil of complexity and uncertainty while it extracts the future (the product) from the simplest activities and imaginations (its natural resource, its raw material).

The politics of security and austerity are but the social and economic implementations of such exposure. And we should add that this constructs the future today towards a post-capitalist society. "There Is No Alternative," the older model's extraction of profits is becoming defunct. 26 It is to be seen if the post-capitalist order will be based on an authoritarian implementation of 'sharing' 27 or the

26 Another point is venture capitalists' reconceptualization of guaranteed basic income as risk capital. Here, Hayekian market ideology fuses with Schumpeterian creative destruction to push back the (welfare) state in favor of an entrepreneurial investor 'society'.

27 E.g.: Social credit scoring applications like the Chinese "Sesame Credit score" are based on derivative logics. Risk options are constantly produced and monitoring others as well as empowerment of new subjectivities and collectives. I therefore conclude with an example of an ambiguous (and even marginal) figure and its environment that at the same time hold the potential for rethinking and reactivating autonomy dynamically and reorients agency beyond critique. As I delineated above, here the aesthetics transform into politics of resolution.

\section{TOWARDS A POLITICS OF RESOLUTION. THE FIGURE OF THE RENEGADE}

"Wall Street is not immoral; it is amoral. When you are not comfortable having an ethical discussion with somebody over lunch, that's a clue. When those types of basic question are taboo you're not going to have much reflection." - Haim Bodek

Scientific and technological progress have had farreaching implications on financial markets, from human to algorithmic market-making; from available time periods for successful investment to those for successful speculation (to discern the terminology for frequencies of transaction turnovers); from value and evaluation to price and pricing, from randomness to risk production as a complex and contingent operation on the future. Beyond the market proper, this shift has radically affected a fundamental category and notion, as Jon Roffe $(2015,29-30)$ argues: "value has no place at all in the market, which is solely the regime of price. This is already implicit in the definition of values as qualitative since the market is a locus for quanta alone. The individual and collective habits and the institutionalized social memory that provide the field in which values come into being and are deployed, is opposed the market, characterized as contingent opening onto the future, and figured in open-ended and unqualified pricing process. While] the market is not a necessary reality - and could not be, given the through-and-through contingent character of the pricing-process - it nonetheless confronts social formations with the radical contingency that the process embodies [...] the pricing process always has the potential to undermine value."

At the same time, the quantification of pricing (beginning with implied volatility calculated on BSM) has affected a flat(er) ontology in financial markets. Especially Ayache and Roffe's recent philosophical elaborations delineate the performative resolution of contingent claims (Ayache's preferred term for derivatives). The hierarchy of underlying price above derivative value has flattened out to the recalibration of derivative prices constantly derived from and implied in the underlying as well as derivatives on derivatives. Volatility of volatility introduces a surface into the market, for which price stands as the non-human non-essentialist actant. The complex and intricate operations and machinations between humans and bots result in new resolutions that either constrain, or, resolve, our perception and cognition. Here, what I term aesthetics of resolution approaches politics of resolution based on a technowledge that conceptualizes and provokes what Levi R. Bryant $(2011,289)$ describes as "an attentiveness [that] would provide us with the resources for thinking strategies of composition that might push collectives into new basins of attraction." And he continues (226-7) with a

oneself recalibrates one's volatile reputation (price) within this technoecology. 
game-changing environment: "Latour proposes the practice of composition as an alternative to critique. Where critique aims at debunking, composition aims at building. Where critique focuses on content and modes of representation, composition focuses on regimes of attraction. If regimes of attraction tend to lock people into particular social systems or modes of life, the question of composition would be that of how we might build new collectives that expand the field of possibility and change within the social sphere." However, as the Flash Crash and its investigations show, in order to push resolution to the level of immediacy, we are in need of an attractor that is both inside and outside the black box. In other words, this counter-agent must provide a trajectory and an exchange out-of and in-to. Black boxes - operators that extract competitive monopolies by folding in a new ontology of resolution - are machines whose performances excise the productive elements from the communicative flow by implementing access hierarchies (including what I called "the rhythual of noise asymmetries"). This undefined reiterated power can only be addressed by the resonance of an agent who not only knows the violence expertly but takes the consequences of exposing herself (embodying the massive risk entailed). I call this attractor-agent the figure of the renegade. Merriam Webster defines "renegade" as a "person who leaves one group, religion, etc., and joins another that opposes it," or, as "someone or something that causes trouble and cannot be controlled." Hence, the renegade is a traitor who transgresses the unwritten laws of complicity and secrecy in her system, her industry. But (often by default rather than design) she becomes educator of the general public (in institutional degrees). This is another point that takes effect in all sectors and fields of power beyond finance: by providing material from undisclosed or classified sources, the renegade has become the principal expert witness for the public, procuring otherwise unavailable (unknown) evidence of information asymmetry (most famously, of course, Edward Snowden).

In the financial context, this particular manifestation of the witness - that does not testify on the basis of real presence - becomes the medium of forensics by a logistics of redirection (e.g. the leaking of confidential material that cannot speak for itself). The story of the Flash Crash offers an example of the significance for the possible production of future publics, depicting in all its complexity the horizon of an exposed and discontinuous self-regulating force against the boundless utopia of a self-regulating market. In the impasse between "perspectives" such as the official investigation and Nanex's renderings, the intricate problem of resolution demonstrates the ambiguity contained: the participation of a traitor is required to unearth data buried in undisclosed documents, in fractions of a second or elsewhere. Hence, the paradigmatic shift to technowledge also gives rise to the cognate notion of a subtly different witness than the eye-witness, one who is capable of challenging calculative violence without plainly being an informant. The financial renegade who presents objects as subjects-of-debate is an expert witness as much as the scientific analyst who subsequently (re)performs the forensic narrative by composing the facts (both can be the same person, or, the incorporation of human and nonhuman expertise). As marginal and ambivalent such moves might sometimes seem they nonetheless constitute opposition within the system. Revisiting Judith Butler's $(1993,163)$ reasoning on the speech act and applying it to our field of inquiry, the one who speaks is directly addressed by the violence: "Insurrectionary speech becomes the necessary response to injurious language, a risk taken in response to being put at risk, a repetition in language that forces change."

When confronted with the black box it seems that composition and association are secondary to the renegade act (they follow it), which itself is secondary to an event or a series of events (violence). Reframing Judith Butler's reading of the performative from the marginal as a potential for insurrection, the renegade opens new inroads into sets of technowledge for building new compositions and collectives by performatively 'inciting' speech from affirmation to allegation. Systemically speaking, a marginalized outside (e.g. the public) can again be $(1993,160)$ "inaugurated into a sociality:" "The performative is not a singular act used by an already established subject, but one of the powerful and insidious ways in which subjects are called into social being from diffuse social quarters, inaugurated into sociality by a variety of diffuse and powerful interpellations. In this sense the social performative is a crucial part not only of subject formation, but of the ongoing political contestation and reformulation of the subject as well. The performative is not only a ritual practice: it is one of the influential rituals by which subjects are formed and reformulated. This point [...] raises again the possibility of a speech act as an insurrectionary act."

The ambivalence, the perils and the counterperformativity of the renegade surface in the flowing story (in: Nestler (2014, min. 40:36): Haim Bodek exposed an order type violation ("Hide Not Slide") by Direct Edge (now a subsidiary of BATS Global Markets) in 2011, which resulted in the record penalty of $\$ 14 \mathrm{~m}$ : ${ }^{28}$ "The whistleblower syndrome is kind of a pattern. The whistleblower says that 'this is obviously wrong and I'm going to call it out' and then when I call it out everyone else is going to realize that it's wrong and it's just going to get fixed right away. What he doesn't realize is that everybody knows about it. So, the message a whistleblower should probably address is [...] 'you know this is wrong and I know all of you recognize this is happening, but this is wrong.' And when you realize that that's what whistleblowing is - that you're making people go through the uncomfortable process of looking at themselves, that's all it is - you realize you're not the hero, you're not bringing new information to the table. You're the guy pointing out the thing that no one wants to see, that everybody knows about. And what's weird about all these cases is that it seems that these, call it injustices, happen in pharmaceuticals, in labor and it's the same pattern over and over, where there's massive injustices which no one wants to talk about and no one wants to admit vocally but everybody knows that's how things work. It doesn't change until the whistleblower does it."

This marginal position notwithstanding, however, the figure of the renegade points to a destination that emphasizes the potential for resistance inside rather than outside a system, in our case financial markets and information capitalism. In fact, the renegade constitutes an act that proceeds from mere dissent (critique within a

28 See: https://www.sec.gov/news/pressrelease/20152.html\#.VLP9qyvF9g0

9 
system) to concrete insurrection (an act of resistance and renunciation). The renegade is an expert acting from a point of no return, a risk taker at the point of ultimate crisis. By speaking out and sharing proprietary data or classified information, she not only discloses what was excluded from public debate but also manifests noncompliance as an act of civil courage. This is a point to be taken seriously even when the experiences that cause her to be disobedient do not inform an ethical decision to act against structural violence, but to an attempt to improve and advance the system - a fact that applies to many industry whistleblowers. The renegade is not a heroic figure; it is as ambiguous as the world she inhabits. But this is not to the disadvantage of the concept: in the midst of (fabricated) noise - in which noise becomes the master of information - the system accidentally yields information; exploiting the event of sectoral asymmetry resolves societal blindness. This renegade act - essentially a violation of current custom, rule or law - produces a host of viable resolution materials across the semantic field of the term ranging from shared visualization, discrimination and cognition to decisionmaking. Whatever the impulse, each act perforates an autonomy that is otherwise decreasingly conceded to natural persons while it is granted to corporate bodies by virtue of their assumed maturity and complexity. Hence, the renegade act reclaims autonomy against all odds and against platform capitalist and data driven forms of sovereignty. That said, it certainly does not constitute political autonomy with a capital A - an autonomy that bestows rights or vests powers. To the contrary, it constitutes a singular act that attracts serious problems and might fail. The renegade is an extremely precarious figure, as history has shown unmistakably.

Moreover, enclosure, virtualization and commodification of data by normalization leads to a constitution of citizenship where the virtual, bot-assembled stake in the subject increasingly separates it from its physical body. What is looming at our political horizon is its disappearance from the social contract and from status, rights and autonomy (Hayles, 1999). But Bruno Latour (1999, 270) reminds us: "While a division between nature and society renders invisible the political process by which the cosmos is collected in one livable whole, the word 'collective' makes this process central." Hence, if we expand the figure of the renegade to a notion of collective voices and practices - a step in the transformation from aesthetics to politics of resolution - we can conceive renegade solidarity as a forceful strategy of renegade incorporations. It counters information capitalism's immediate biopolitical grip on life and its ever-shrinking distance (physically, affectively and ethically), which it manufactures from the absolute distance it invests in - knowledge does not resolve, it dissolves into what it cannot know but what it can intensify as price.

The making of resolution and the renegade act stand against the violence of information asymmetries and noise by counter-constructing their "assemblies." Renegade solidarity stands for a politics of resolution of counterinstitutions, in which a collective of experts (activists, scientists, artists, nonhuman agents) act together to enhance resolution across the whole gamut of its semantic and political meaning. ${ }^{29}$ The autonomy gained is ambivalent, marginal, in a state of constant flux and highly volatile if not outright dangerous. At the same time, it creates value by producing myriad forms of knowledge and generating tactics of infiltration. In intensifying, reinforcing recursive acts that belong to language as well as to other logics of expression, new modes of making (poietics) can come into existence and produce new ways of perceiving, thinking and making the world. This is a case of offering platforms of affiliation (rather than the conformity exploited by platform capitalism); and a case of strengthening the desire and urgency to participate in forms of common ownership and autonomies that exist side by side and in flux. ${ }^{30}$ And in which the future is seen with differentiating, envisioning and resolving eyes through all the folds that distort the playing field. In short, to move from low-res to high-res across the whole gamut of resolution's consequential - and hence not only technological or financial but also philosophical and political - meanings.

\section{CONCLUSION}

The term resolution speaks of intellectual, physical and affective potentials; it involves technological and algorithmic properties, operations and the "distances" between them. It is a socially powerful node of how we may sense, map, differentiate and support material and at the same time performative relations. Moreover, it holds the potential for thinking and creating access to value(s) in radical contrast to the capitalist price(ing) engine and its proprietary logic, without losing the performative edge necessary within complex societies in flux. Against the enclosures and the noise of capital, autonomy is to be reconceptualized as a dynamic, open, aleatoric and instantaneous process (acts connected to a multitude of contingent moments) that purposely integrates ambiguous, heteronomous influences in order to make resolution in the full meaning of the term. The multifaceted semantic field of resolution and its technological, aesthetic, social and political significance - ranging from visualization, discrimination, intelligence and knowledge to intention, purpose, (common) initiative and (joint) decision-making - offers a collectivity that presents a conceptual basis for a practice, which does not lose sight of rethinking socio-political constitutions against the conditions that make the ruptures and breaches of social contracts possible in the name of proprietary interest. Thus, it plays an eminent role in the effort of tracing aesthetic (what we 'see'), poietic (what we 'make') as well as political (how we apply these to resolution as decisionmaking) consequences. Carving out an aesthetics of resolution in order to move towards a politics of resolution is a move from dissent to insurrection in solidarity with the figure of the renegade. Here, solidarity can either mean becoming or supporting the traitor-educator. Here, we encounter a revolutionary figure of our time that "re-maps" the playing field within a emancipatory paradigm that is not only interested in research and analysis of data and events (critique) but in the technopolitical consequences

as regards homosexuality might be helpful also in the context of systemic as well as individual implications for renegade solidarity in that "we surely need to take seriously the contention that 'coming out' is intended as a contagious example, that it is supposed to set a precedent and incite a series of similarly structured acts in public discourse."

30 Including open source apps and infrastructures as well as blockchain technologies such as cryptocurrencies and contracts. 
of data processes, evaluations and decisions in order to make the black box speak and change its course (insurrection-resistance).

\section{ACKNOWLEDGMENTS}

l'd like to thank Haim Bodek for his insights and engagement. This research started with the exhibition Forensis at Haus der Kulturen der Welt, Berlin, 2014, curated by Anselm Franke and Eyal Weizman (a project by Forensic Architecture and the Centre for Research Architecture, Goldsmiths, University of London).

\section{REFERENCES}

Appadurai, A. (2015) Banking on Words. The Failure of Language in the Age of Derivative Finance. Chicago: The University of Chicago Press.

Appadurai, A. (2016) The Wealth of Dividuals. In: B. Lee, R. Martin; Derivatives and the Wealth of Societies. Chicago: The University of Chicago Press.

Ayache, E. (2015) The Medium of Contingency. An Inverse View of the Market. Basingstoke: Palgrave Macmillan.

Black, F. (1986) Noise. Journal of Finance, 41 (3), 529-543.

Bryant, L. R. (2011) The Democracy of Objects. London: Open Humanities Press.

Butler, J. (1993a) Bodies that Matter: On the Discursive Limits of "Sex. New York, London: Routledge.

Butler, J. (1997b) Excitable Speech. A Politics of the Performative. New York, London: Routledge.

Callon, M. (2006) What does it mean to say that economics is performative?. CSI working papers series 005 .

CFTC/SEC (2010) Reports of the staffs to the joint advisory committee on emerging regulatory issues. September 30.

Coeckelbergh, M. (2015) Money Machines. Electronic Financial Technologies, Distancing, and Responsibility in Global Finance. Farnham: Ashgate.

Deleuze, J. (1994) Difference and Repetition. New York: Columbia University Press

Farmer, J. D. (2013) The impact of computer based training on systemic risk. London School of Economics, January 11.

Hayles, N.K. (1999) How we Became Posthuman. Virtual Bodies in Cybernetics, Literature, and Informatics. Chicago: The University of Chicago Press.

Ho K. (2009) Liquidated: An Ethnography of Wall Street. Durham: Duke University Press.
Kirilenko, A., Kayle, A.S., Samadi, M., Tuzun, T. (2011) The Flash Crash: The Impact of High Frequency Trading on an Electronic Market. Online: http://www.cftc.gov/idc/groups/public/@economicanalysis/do cuments/file/oce_flashcrash0314.pdf

Knight, F.H. (1921) Risk, Uncertainty, and Profit. Boston: Hart, Schaffner \& Marx: Boston.

Latour, B. (1993a) The Pasteurization of France. Cambridge: Harvard University Press.

Latour, B. (1999b) Pandora's Hope: Essays on the Reality of Science Studies. Cambridge: Harvard University Press.

Li Puma, E. (2016) Ritual in Financial Life. In: B. Lee, R. Martin (Eds.), Derivatives and the Wealth of Societies. Chicago: The University of Chicago Press.

MacKenzie, D. (2016) A Material Political Economy: Automated Trading Desk and Price Prediction in High-Frequency Trading. Social Studies of Science, 47 (2), 172-194.

MacKenzie, D. (2006) An Engine, not a Camera. Cambridge: MIT Press.

McLuhan, M (1964) Understanding Media: The Extensions of Man. Cambridge: MIT Press.

Meerman, M. (2013) The Wall Street Code. Film documentary, 51 min. Online: http://www.youtube.com/watch?v=kFQJNeQDDHA.

Nanex (2010) Flash Crash Summary Report. Sept. 29. Online: http://www.nanex.net/FlashCrashFinal/FlashCrashSummary. html

Narang, R. K. (2014) The Truth about High-Frequency Trading. Hoboken: Wiley.

Narang, R. K. (2015) Inside the black box: the simple truth about quantitative trading. Hoboken: Wiley.

Nestler G. (2014) Contingent Ethics. Portrait of a Philosophy series II: Haim Bodek. Single channel video. Online: https://vimeo.com/channels/AoR

Roffe, J. (2015) Abstract Market Theory. Basingstoke: Palgrave Macmillan.

Simmel, G. (1990) The Philosophy of Money. London: Routledge.

Taggart, A. (2012) Eric Hunsader: Investors Need to Realize the Machines Have Taken Over. The Blink of an eye is a lifetime for HFT algos. Peak Prosperity, October 6.

Wilkins, I., Dragos, B. (2013) Destructive Destruction? An Ecological Study of High Frequency Trading. Mute, January 22. 